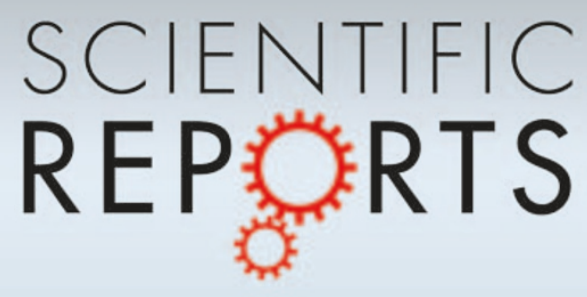

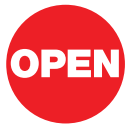

SUBJECT AREAS:

ATOMIC AND MOLECULAR PHYSICS

THEORETICAL PHYSICS

CONDENSED MATTER PHYSICS

QUANTUM PHYSICS

Received

1 December 2011

Accepted

2 March 2012

Published

12 April 2012

Correspondence and requests for materials should be addressed to A.D. (arnabdas@pks. mpg.de)

\title{
Winding up superfluid in a torus via Bose Einstein condensation
}

\author{
Arnab Das ${ }^{1}$, Jacopo Sabbatini² \& Wojciech H. Zurek'
}

'Theory Division, LANL, MS-B2 13, Los Alamos, NM 87545, USA, ${ }^{2}$ ARC Centre of Excellence for Quantum-Atom Optics, School of
Mathematics and Physics, University of Queensland, Brisbane, QLD 4072, Australia.

Phase transitions are usually treated as equilibrium phenomena, which yields telltale universality classes with scaling behavior of relaxation time and healing length. However, in second-order phase transitions relaxation time diverges near the critical point ("critical slowing down"). Therefore, every such transition traversed at a finite rate is a non-equilibrium process. Kibble-Zurek mechanism (KZM) captures this basic physics, predicting sizes of domains - fragments of broken symmetry - and the density of topological defects, long-lived relics of symmetry breaking that can survive long after the transition. To test KZM we simulate Bose-Einstein condensation in a ring using stochastic Gross-Pitaevskii equation and show that BEC formation can spontaneously generate quantized circulation of the newborn condensate. The magnitude of the resulting winding numbers and the time-lag of BEC density growth - both experimentally measurable - follow scalings predicted by KZM. Our results may also facilitate measuring the dynamical critical exponent for the BEC transition.

econd order phase transitions are always associated with divergence of both the relaxation time ("critical slowing down") and healing length ("critical opalescence") at the critical point. These divergences imply inevitable non-adiabaticity when a system is driven across the transition and result in a finite size of domains that can coordinate symmetry breaking. That size is set by the healing length at the time when the system can no longer keep up with the externally imposed change of parameters that drive it through the transition, and adiabatic following gives way to impulse "freezeout" of its state.

This mosaic of domains with independent choices of broken symmetry may result in topological defects. Their densities at formation will bear universal signatures of the underlying phase transition. Kibble-Zurek mechanism (KZM) is the theoretical framework that describes the dynamics of symmetry breaking in second order phase transitions by taking into account finite speed of propagation of the relevant information ${ }^{1-5}$. KZM leads to a quantitative estimate for the density of defects ${ }^{3-6}$. In particular, it predicts that their density should scale with the quench rate. The scaling exponent predicted by KZM is a function of the critical exponents of the underlying equilibrium phase transition. The basic ingredients for this prediction are the critical exponents - i.e., the universality class of the transition. Therefore, applicability of KZM spans physical phenomena of enormous variety and scales, starting from microscopic phenomenon of vortex formation in superfluid, right up to phenomena of astronomical scales, like formation of structures (cosmic strings, monopoles, etc.) in the early Universe.

Kibble-Zurek mechanism has been tested in a number of primarily numerical studies, see, e.g., Refs. ${ }^{7-14}$. However, on the experimental side, while, as of now, data confirm key qualitative predictions of KZM (creation of topological excitations) $)^{15-25}$, its key quantitative prediction (scaling of their density with the rate of quench) has not yet been convincingly demonstrated (see, however, Refs. ${ }^{23-25}$, for suggestive indirect evidence). The difficulty involves controlling sufficient range of quench time scales as well as counting defects.

In this article we pave a way around this long-standing hindrance. Within the setup of Bose-Einstein condensation transition in an effectively one-dimensional ring, we demonstrate that KZM scaling laws can be observed involving stable and experimentally accessible quantities, namely, the variance of the winding number $W$ and the non-adiabatic response time for density growth. Looking for signature of KZM in growth of bulk density is a novel perspective which allows bypassing the traditional difficulty of counting and resolving defects. Our demonstration is also a long overdue numerical verification of the original prediction proposed within the setup of superfluid circulation in an annulus ${ }^{3}$.

Manifestations of symmetry breaking associated with BEC formation are spectacular and diverse $e^{6-10,12,13,15,17-19,26}$. Formation of BEC breaks the $U(1)$ symmetry of the phase of the condensate wave-function. We show that when BEC forms in a ring cooled through the critical temperature, this symmetry breaking may result in spontaneous rotation in the newborn condensate. Persistence of the resulting flow is assured by topological stability of the 
quantized winding number $W$. We show that $W$ s stabilize soon after the transition, and the variance of their distribution follows the scaling predicted by the KZM. Density growth of BEC also exhibits behavior consistent with KZM.

Recently, persistent circulation of BEC in toroidal trap has been achieved experimentally by stirring the cloud ${ }^{27}$. In particular, with the advent of circular trapping potential for $\mathrm{BEC}^{28-31}$, the possible experimental testing of KZM is around the corner. Density growth in $\mathrm{BEC}$ formation with variable cooling rate has also been observed ${ }^{15,32}$. We show that a similar setup would allow both the testing of density growth scaling and determining the critical properties of the BoseEinstein condensate $e^{3-5}$.

\section{Results}

The Model. We consider a BEC in a quasi-1D ring of circumference $C$, an idealization of quasi-1D toroidal geometry, e.g., see $\mathrm{se}^{28-31,33,34}$. We model it using the stochastic Gross-Pitaevskii equation (SGPE) ${ }^{35-37}$ :

$$
(i-\gamma) \frac{\partial \phi}{\partial t}=-\frac{1}{2} \frac{\partial^{2} \phi}{\partial x^{2}}+\epsilon(t) \phi+\tilde{g}|\phi|^{2} \phi+\eta(x, t),
$$

where $\phi=|\phi(x)| e^{i \theta(x)}$ is the condensate wavefunction and $\eta(x, t)$ is the thermal noise satisfying the fluctuation-dissipation relation $\langle\eta(x$, t) $\left.\eta^{*}\left(x^{\prime}, t^{\prime}\right)\right\rangle=2 \gamma T \delta\left(x-x^{\prime}\right) \delta\left(t-t^{\prime}\right)$, with $\gamma$ representing the dissipation, $T$ the noise amplitude, $\tilde{g}$ the non-linearity parameter and $-\epsilon$ the chemical potential. We use dimensionless units given by $\hbar=1, m=1$ (mass of a ${ }^{87} \mathrm{Rb}$ atom) and unit of time $=1 / \omega_{0}$ with $\omega_{0}=200 \times 2 \pi \mathrm{Hz}$. We set $\tau_{0}=\gamma^{-1}+\gamma$ and $\xi_{0}=1 / \sqrt{2^{7,8}}$.

Leaving aside the noise and dissipation, the above system can be described by the energy functional $\mathcal{E}=\oint_{\text {ring }}\left[\frac{1}{2}\left|\partial_{x} \phi\right|^{2}+U(|\phi|)\right] d x$, where $U(|\phi|)=\epsilon|\phi|^{2}+\frac{1}{2} \tilde{g}|\phi|^{4}$. Extremizing the energy functional we obtain $\phi=0$ for $\epsilon>0$ and $\phi=\sqrt{|\epsilon| / \tilde{g}} \exp (i \theta)$ for $\epsilon<0$, where $\theta$ is the wave function phase ( $\epsilon=0$ is the critical point). We induce the transition by quenching $\epsilon$ :

$$
\epsilon(t)=-t / \tau_{Q}
$$

from an initial $\epsilon>0$ to a final $\epsilon<0$, and allow the system enough time to stabilize. The critical point is crossed at $t=0$. Simulation of cooling of BEC by quenching chemical potential within the framework of SGPE has been shown to reproduce experimental results on defect (vortex) generation; see, e.g. Refs. ${ }^{15,35-37}$.

KZM in 1-D BEC Ring. When BEC is formed via cooling through the critical point, non-adiabaticity is enforced by diverging relaxation time $\tau$ and healing length $\xi^{3-5}$ :

$$
\tau=\tau_{0} /|\epsilon|^{v z} ; \quad \xi=\xi_{0} /|\epsilon|^{v} .
$$

Here $v$ and $z$ are the critical exponents, $\xi_{0}$ and $\tau_{0}$ are determined by the microscopic details of the system. According to KZM, as $\epsilon$ approaches 0 , after the instant $-\hat{t}$ the relaxation time $\tau(-\hat{t})$ exceeds the timescale $\mid \epsilon / \dot{\theta}_{t=-\hat{t}}$ of the change imposed by quench, and the state of the system freezes. Its order parameter behaves impulsively (i.e., remains effectively frozen) within an interval between $\pm \hat{t}$ and starts dynamical evolution again thereafter. KZM gives

$$
\tau(\epsilon(-\hat{t}))=|\epsilon / \dot{\epsilon}|_{t=-\hat{t}}=\hat{t} .
$$

For the linear quench in Eq. (2), we get from Eqs. (3), (4)

$$
\hat{t}=\left|\tau_{0} \tau_{Q}^{v z}\right|^{1 /(1+v z)}, \quad \hat{\xi}=\xi_{0}\left|\tau_{Q} / \tau_{0}\right|^{v /(1+v z)},
$$

where $\hat{\xi}=\xi(\hat{t})$. As $\epsilon(t)$ becomes negative, condensate starts forming with a phase profile $\theta(x)$ consisting of random patches: phase is approximately uniform over the length-scale $\hat{\xi}$. Within each such patch, its value is chosen independently and randomly (different stages of condensate formation in a uniform ring are shown in Fig. 1 upper row). Therefore, we can estimate the variance of the total phase $\theta_{C}=\oint_{\text {ring }} d \theta(x)$ within a torus of circumference $C$ by
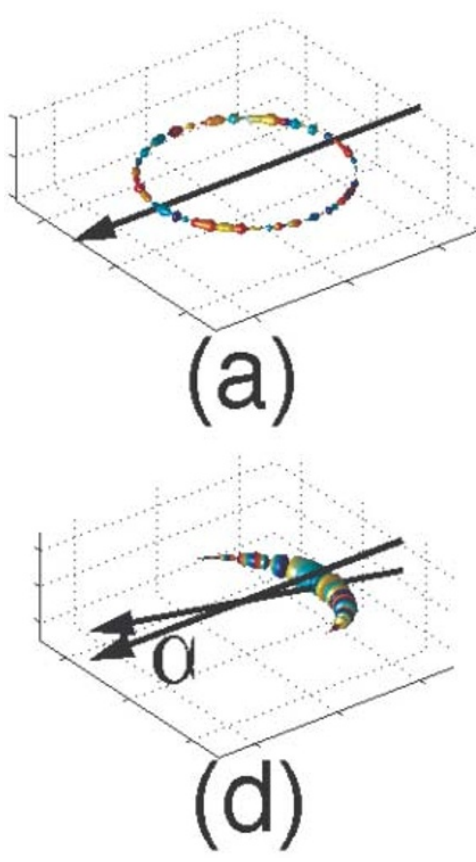

Figure 1 Condensate Growth in the ring. Sequences of isodensity surfaces for growing condensate for a single quench realization with $\tau_{Q}=0.01$ for flat ring (top line, time flowing from (a) to (c)) and tilted ring with tilting angle $\alpha=3^{\circ}$ (bottom line, time flowing from from (d) to (f)). Color represents the phase of the condensate along the ring, showing magnitude of $W$ to be 1 and 2 respectively in the flat (c) and the tilted (f) rings. The 3D equivalent is constructed for a ring with isotropic harmonic transverse confinement with frequency $\omega_{0}=200 \times 2 \pi \mathrm{Hz}$ and other parameters as mentioned in the text for Figs. 2 and 3 (see "Methods" section for discussion on this equivalence). Formation of random equi-phased patches at the onset of condensation is clearly visible. 

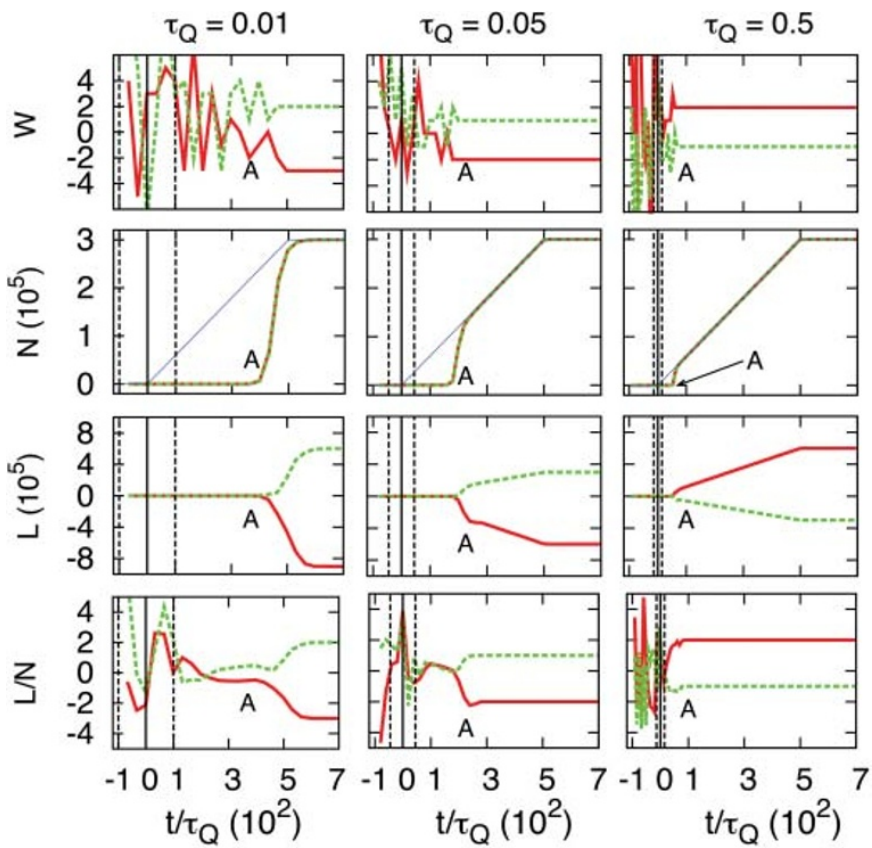

Figure $2 \mid$ Quenching into a BEC and its consequences. Time evolution of the winding number $W$, total number of BEC atoms $N$, total angular momentum $L$ and specific angular momentum $L / N$ for random instances of quench realizations with different $\tau_{\mathrm{Q}}$. The quench is induced by ramping $\epsilon$ linearly from -500 to +500 in each case (Eq. 2). For each $\tau_{Q}$, different quantities for the same realization are plotted in same color. The black vertical lines indicates $t_{c}=0$ (continuous, middle one) and the two on either sides (dashed) indicate $\pm \hat{t}$, where $\hat{t}=\sqrt{\tau_{Q} \tau_{0}}$, and $\tau_{0}=\gamma^{-1}+\gamma$. The second row shows the adiabatic-impulse transition in BEC growth. While $\hat{t}$ gives the KZM estimate for the impulse to adiabatic transition boundary, the instant denoted by $A$ in the figure marks the point where $N$ shoots up sharply. The thin blue lines indicate the equilibrium values of $N$ for the corresponding instantaneous values of $\epsilon$. Prior to the quench, we thermalize the system at the initial value of the chemical potential. $T=10^{-3}, \gamma=10^{-2}$ and $\tilde{g}=0.05$ are held constant for all time.

considering the sum of $\sim C / \hat{\xi}$ uniform random variables, each having the same variance $\pi^{2} / 3$ ( $\theta$ taking any value between $\pm \pi$ ). This implies Gaussian distribution for $\theta_{c}$ (in agreement with inset of Fig. 3a) with variance $\sigma^{2}\left(\theta_{c}\right)=\left\langle\theta_{c}^{2}\right\rangle=\left(\pi^{2} / 3\right)(C / \hat{\xi})$ when averaged over many realizations. As the wave function is singlevalued, we must have $\theta_{c}=2 \pi W$, where $W$ is the integer winding number. So, Eq. (5) predicts dispersion:

$$
\sigma(W)=\sqrt{\left\langle W^{2}\right\rangle}=\frac{\tau_{0}^{1 / 8} \xi_{0}^{-1 / 2}}{2 \sqrt{3}}\left(\frac{\sqrt{C}}{\tau_{Q}^{1 / 8}}\right)=\sqrt{\frac{\pi}{2}}\langle|W|\rangle,
$$

using the mean field exponents $z=2$ and $v=1 / 2^{7,8}$.

Spatial gradient of $\theta(x)$ gives local flow velocity. Therefore, $W$ quantifies the net quantized circulation of the condensate around the ring. Thus, breaking of $U(1)$ symmetry leads to independent phase selection and results in a net superfluid circulation in the ring $^{3-5}$. The scaling predicted above is valid when expected $|W|>$ 1. It steepens for $\langle|W|\rangle \sim 1$ or smaller; $\operatorname{see}^{23-25,38,39}$ and references therein.

We also observe that the scaling of $\hat{t}$ in Eq. (5) can be extracted from the growth of the condensate density. It shows a sharp "knee" behavior, revealing a characteristic response time as the transition point is crossed. The response time gives an effective $\hat{t}$, which follows the scaling in Eq. (5).

Simulation Results for Winding Numbers. We integrate Eq. (1) numerically to study the quench dynamics. Evolution of $W$, BEC particle number $N$, total angular momentum $L$ and specific angular momentum $L / N$ are illustrated in Fig. 2. We define total angular momentum $L$ through single particle angular momentum operator. Assuming our wavefunction $\phi(x)=\sqrt{N} \phi_{\text {single }}(x)$, the total angular momentum (only the z-component is non-zero) is given by $L=i \frac{C}{2 \pi} \oint_{C} \phi^{*}(x) \frac{\partial \phi}{\partial x} d x$. Each quench in this figure continues from $\epsilon=500$ to $\epsilon=-500$, where it is held fixed for the rest of the time. Though the flow involves more mass as $\epsilon$ decreases, $W$ acquires a stable value right after the symmetry-breaking. Both $L$ and $N$ grow as long as $\epsilon$ decreases, but $L / N$ stabilizes to a steady value along with $W$. This is analogous to the build up of persistent current in superfluid $\mathrm{He}^{3-5,40}$. For spatially uniform density the simple relation $L / N=W$ holds and we observe that at the tail-end of the quench (last row), when the density fluctuations are ironed out due to dissipation. Final kinetic energy of the BEC depends on the final steady value of $W$ and scales with it quadratically. $W$ stabilizes (first row) in the wake of the condensation, when $N$ (second row) is still negligible. Thus, $W$ retains phase information from soon after BEC formation at the time of symmetry breaking. Once stabilized, $W$ is resilient to dissipation and typical ambient thermal fluctuations, due to its quantized nature. The scaling in Eq. (6) is verified by averaging $|W|$ over $>10^{3}$ realizations. Here the quenches are also between $\epsilon= \pm 500$, except for the fastest one, for which it is done between $\epsilon= \pm 1000$, so that the quench begins and ends outside the impulse region between $\pm \hat{\epsilon}$. The simulation results compare favorably with the KZM prediction, as summarized in Fig. 3(a). A direct comparison
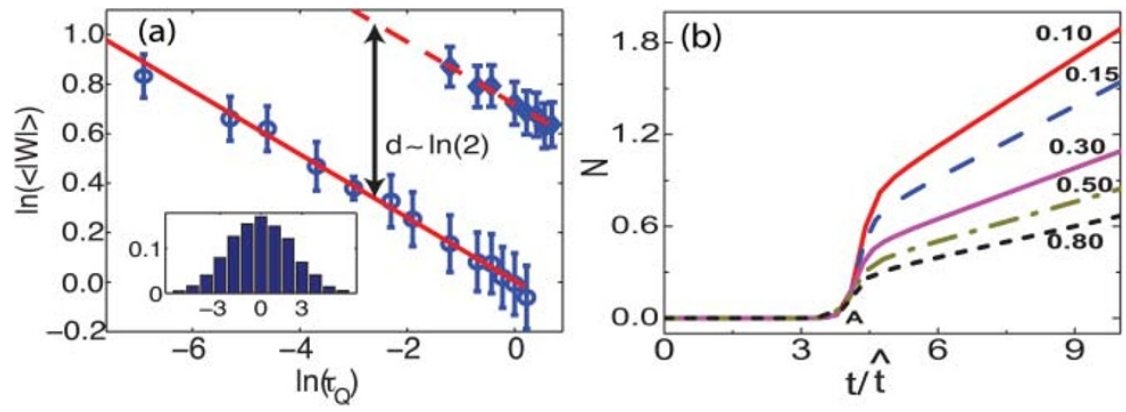

Figure $3 \mid$ Scaling Laws. (a) The scaling for $\langle|W|\rangle=\sqrt{2 / \pi} \sigma(W)$ (averaged over $>10^{3}$ realizations) with $\tau_{Q}$ for $C=30$ (open circle) and 120 (filled diamonds). The error bars indicate the standard deviations. Best fits (red line) in the Fig. give exponents $0.1262 \pm 0.0026$ for the $C=30$ and $0.126 \pm$ 0.0075 for $C=120$, compared to the expected $v / 2(1+v z)=1 / 8=0.125$ (Eq. 6). Random walk in phase prediction $\langle|W|\rangle \sim \sqrt{C}$, i.e., $\log \left(\langle|W|\rangle_{C=120}\right)-$ $\log \left(\langle|W|\rangle_{C=30}\right) \sim \log 2$ (Eq.2), is also verified (the difference shown in the Fig. is $\approx 0.308$ compared to $\log 2 \approx 0.301$ ). The inset shows histograms for distribution of $W$. (b) $N$ vs $t / \hat{t}$. The "knees", where $N$ shoots up (point A), show perfect overlap for different $\tau_{Q}$ 's (values marked in the Fig.), and can be used for estimating critical exponents. All static parameters are same as in Fig. 2. 
between the numerical results and KZM formula for our model (Eq. 6) yields, $\sigma(W)(\mathrm{KZM})=5.94,4.86$ and 3.65 versus $\sigma(W)$ (numerical) $=2.33,1.83$ and 1.35 for $\tau_{Q}=0.01,0.05$ and 0.5 respectively. With "naive" KZM (choices of phases over $\hat{\xi}$-sized regions are completely independent), mismatch of this order is consistent with (actually, significantly less than) past observations ${ }^{12,13,41}$. We shall comment on this further in the Discussion. The square-root dependence of $\langle|W|\rangle$ on $C$, Eq.(6) is also verified there, as the values of $\langle|W|\rangle$ obtained for $C=120$ are approximately double of those obtained for $C=30$ for the same $\tau_{Q}$ s (Fig. 3a).

Thorough initial thermalization is crucial in producing equilibrated initial state (and, hence, physically motivated final state). In particular, noise should randomize the phase along the entire circumference $C$. Otherwise, winding numbers saturate (as we have observed with runs that did not attain this initial long-range thermalization of the phase). This randomization of the phase is in effect a diffusive process that requires time $\sim C^{2}$, which renders accurate reproduction of the scaling behavior for large $C$ numerically costly.

Adiabatic-impulse transition is at the heart of the scaling laws predicted by KZM. Here we observe for the first time its manifestation in BEC formation as a scaling-law for the length of the impulse reaction time (effective $\hat{t}$; see, e.g. Ref. ${ }^{41}$ ) for the condensate density growth (see however, similar plot for time-dependent spin alignment in spinor $\mathrm{BEC}^{42}$ ). This is an important result, since density evolution in a condensation process is easier to study experimentally than the phase ordering associated with defect dynamics. We compare (Fig. 2, second row) the growth of $N$, with the instantaneous equilibrium value of $N$ (thin blue straight-line segments), obtained from the relation $\epsilon=-\tilde{g} N / C$ (valid for small $\tilde{g}$ ). Initially, $\epsilon>0$, and $N$ is negligible. After crossing $t_{c}$ the instantaneous equilibrium value of $N$ increases linearly with the rate $\dot{N}=C / \tilde{g} \tau_{Q}=0.6 \times 10^{5}$ till $t=$ $500 \tau_{Q}$, where the ramp is stopped and equilibrium value for $N$ settles to $3 \times 10^{5}$. But for the actual evolution, the length of the period from $t$ $=t_{c}=0$ up to the point denoted by $A$ (a sharp knee) in respective figures (see also Fig. $3 \mathrm{~b}$ ), should be proportional (and of the order of) $\hat{t}$ and - in our SGPE case - should scale as $\tau_{Q}^{1 / 2}$. Our results confirm this scaling, as shown by the overlap of $N$ around the knee, when plotted against $t / \hat{t}$ for different $\tau_{Q}$ (Fig. 3b). After this knee point, $N$ catches up rapidly with its equilibrium value. $W$ and $L / N$ also stabilize around $A$.

Unlike other BEC relics of symmetry breaking (e.g., solitons), which are difficult to resolve due to thermal noise, and decay due to dissipation, $W$ bears a very stable and readable signature of the underlying phase transition due to its topological stability and integer nature. Statistics of $W$ can be presumably studied, e.g. within the experimental setups such as $^{27-31,33,34}$. Experimental study of the growth of BEC density with adjustable cooling rate parameter has been reported already by Esslinger group ${ }^{32}$ (cooling by sudden ramp of confining RF field), and within toroidal geometry by Anderson group $^{15}$ (both with sudden and linear ramp of RF field). They observed the linear growth regime, as well as the "knee" feature that provides an experimental counterpart of the effective $\hat{t}$. This should allow direct verification of the KZM scaling law and quantitative determination of the exponent for $\hat{t}$. Moreover, experimental determination of the dynamical exponent $z$ may be possible employing the KZM formulae $v / 2(1+v z)$ and $1 /(1+v z)$ for the measured values of $\langle|W|\rangle$ and effective $\hat{t}$ exponents, Fig. 3(a) and 3(b), respectively, and solving for $v, z$. The scaling law involving $N$ may be amenable to more accurate experimental determination, since the exponent for $\hat{t}$ scaling $\left(\hat{t} \sim \tau_{Q}^{1 / 2}\right)$ is larger than that for the scaling of $\left(\sigma(W) \sim \tau_{Q}^{-1 / 8}\right)$. Note that for real BEC transition in $3 \mathrm{D}$, theoretical prediction is $v=$ $2 / 3$ (close to experimental value, $0.67 \pm 0.13,{ }^{44}$ ) and $z=3 / 2$. Finally, the sample to sample fluctuations in the growth of $N$ are much smaller than those of $W$ (see Fig. 2), and hence requires less averaging over realizations.

\section{Discussion}

Our simulations reproduce scalings of the winding number and $\hat{t}$ predicted by KZM, but the "naive" random walk argument including Eq. (6) we have used to estimate $\langle|W|>$ results in an overestimate by a factor $f \sim 2.5$. This discrepancy deserves a comment both because it is there and because it is surprisingly small: Factors $f \sim$ 10 were reported in the past ${ }^{12,13,41}$.

We first note that KZM estimate - one $\hat{\xi}$-sized defect fragment per domain of size $\hat{\xi}$ - is general, and this generality is obtained at the price of focusing on what is dominant (scalings) and ignoring (subdominant) microphysics. For instance, it is clear that the estimate of $\hat{\xi}$ will depend on the noise temperature and on the nonlinearity parameter. Moreover, density of defects will depend on their nature, and there are systems (such as ${ }^{3} \mathrm{He}$ ) where there are many different types of defects that can be created by the same transition, and there is no reason for their densities to be the same.

Detailed studies show that such additional inputs affect density estimates but are either subdominant (e.g., there is a logarithmic dependence on noise and nonlinearity, as can be expected from the nature of the instability after the quench ${ }^{43}$ ) or are too complicated for precise treatment $\left({ }^{3} \mathrm{He}\right)$. However, these additional inputs do not change scaling of $\hat{\xi}$, and therefore, scaling of the number of topological defects with the quench rate.

This focus on scalings is very much in the spirit of the renormalization approach to second order phase transitions: Microphysics sets dimensionfull inputs that determine healing length and relaxation time, but scalings of these two quantities near the critical point are independent of microphysical details. This allows for the classification of second order phase transitions in terms of critical exponents. Similarly, KZM predicts scalings, but only estimates prefactors.

In contrast to past numerical experiments (where defect separations were typically $f \hat{\xi}$, with $f \sim 10$ ) we have seen KZM estimates of winding numbers only a factor of about 2.5 larger than the numerical estimates. This improved accuracy is because - in contrast to past exercises of this sort - in the constrained problem we have addressed we were able to be more precise in taking "SGPE microphysics" into account. Thus, we have estimated that domains of size $\hat{\xi}$ will have phases that differ by $\pi / \sqrt{3}$, and the geometry of the problem allowed us to avoid some of the difficult questions concerning dynamics of the post-quench phase ordering. However, these inputs are still only estimates: It is clear from Fig. 1 that different "beads" of the newly formed BEC do not have the same size. Moreover, it is quite likely that early on - around the time when the condensate begins to encompass the whole torus - winding number can change (see Fig. 2). Last not least, the instant when this seems to happen scales in accord - but does not coincide - with the estimate of $\hat{t}$.

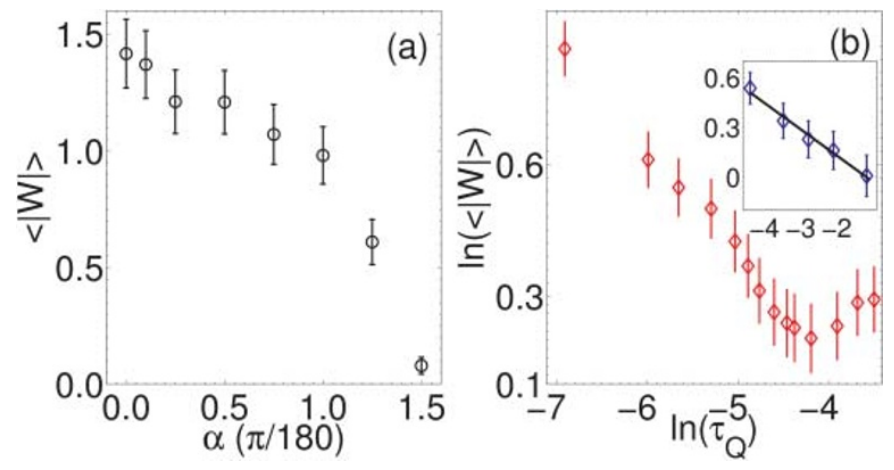

Figure $4 \mid$ Winding up a tilted BEC ring. (a) $\langle|W|\rangle$ vs $\alpha$ for $\tau_{Q}=0.1$ (other parameters same as in Fig. 2). (b) Suppression of scaling behavior of $\langle|W|\rangle$ with $\tau_{Q}$ for $\alpha=1^{\circ}$. The inset shows scaling behavior for smaller tilt of $\alpha=$ $0.5^{\circ}$ with a larger exponent $0.158 \pm 0.011$. 
We conclude this part of the discussion by noting that KZM is a general theory of the dynamics of second order phase transitions, but that - like the universality classes on which it is based - it largely ignores microphysical details and does not aim to predict dimensionfull prefactors. Thus, while a more careful estimate of the expected magnitudes of winding numbers are possible in the simple model we have examined, the real aim of KZM and a good way to test it is to verify that it correctly predicts scalings of the topological relics of the transition from the critical exponents (which, for the real BEC, are anyway different than in the case of our SGPE model). Toroidal BEC traps are needed for this, and they are not the "popular model". Fortunately, our study suggests an alternative, more limited but also potentially more accessible possibility - testing for the scaling of delay in BEC density growth.

Predictions of KZM can be substantially perturbed by natural sources of experimental imperfections in the form of inhomogeneity in the potential. An overall non-uniformity may have many causes. To be specific, we shall think of tilting the torus in the gravitational field. This is represented by an additional potential of the form $V(\vartheta)=m g \sin (\alpha) R[1-\cos (\vartheta)]$ where $\alpha$ represents the tilting angle, $\vartheta=x / R$ is the angular coordinate denoting the position on the ring, $R$ $=C / 2 \pi$ and $g$ the gravitational acceleration. Different stages of condensate formation in the tilted ring are shown in Fig. 1 (lower row). Fig. 4(a) shows suppression of $W$ as the function of $\alpha$. Inset of Fig. 4(b) shows that the scaling behavior still persists for very small tilting of $\alpha=0.5^{\circ}$, but the exponent increases. The main Fig. 4(b) shows the disappearance of the KZM scaling for slightly bigger tilting $\left(\alpha=1^{\circ}\right)$. Suppression of $W$ could be caused in the tilted ring due to the competition between the finite velocity $v_{F}$ of the critical front (determined by the critical condition set locally by the inhomogeneity $\left.^{6}\right)$ and the relevant sound velocity $\hat{v}=\hat{\xi} / \tau(\hat{t})$ at which the correlation is established between the condensate phase. Formation of phase-inhomogeneity (local flow) is suppressed wherever $\hat{v}>v_{F}$, since phase correlation is maintained throughout the condensation process there: the newborn condensate selects the same phase as the existing condensate due to local free-energy minimization ${ }^{6,45,46}$. This implies steeper fall of $\langle|W|\rangle$ with $\tau_{Q}{ }^{6}$. But in our case, the above condition is not met for $\alpha=0.5^{\circ}$. The same criteria also cannot explain the suppression of $W$ for $\alpha=1^{\circ}$ (Fig. 4b). One possible explanation of this suppression may lie in the dissipation of kinetic energy prior to the formation of the complete BEC ring. The local flows generated by KZM at the bottom of the ring are susceptible to dissipation before the topological constraint (that protects the quantized circulation) is imposed, i.e., before the BEC is formed completely up to the ring top. Such early energy loss might leave the condensate with kinetic energy insufficient for quantized circulation. We note that tilting a BEC ring experimentally and applying that to offset inadvertent horizontal misalignment has been achieved recently ${ }^{34}$, so while we have not developed the theory of various imperfections (exemplified here by tilting in the gravitational field), control with the accuracies that may compensate for (or avoid) such problems seems possible.

For very slow quenches (large $\tau_{Q}$ ), however, $|W|$ doesn't vanish in the tilted case, as the density doesn't grow on the top till the critical front reaches the top, and topological protection doesn't apply till then. If this period is long enough, thermal fluctuations may create sufficient random walks in phase to induce a net circulation by the time the density becomes significant everywhere. The resulting circulation may hence even grow with $\tau_{Q}$ in this regime, as seen from the Fig. 4 .

To summarize, we showed that temperature quench through the critical point can produce spontaneous circulation of BEC in a ring and verified long standing scaling predictions of $\mathrm{KZM}^{3}$. Our demonstration involving winding number and condensate density paves a way for experimental verification of KZM scaling laws. It also provides prescription for experimental determination of the critical exponents of the underlying BEC transition.

\section{Methods}

We simulate the quasi-1D BEC by integrating 1-D Stochastic Gross Pitaevskii equation (Eq. 1) numerically using 4th order adaptive step-size Runge-Kutta algorithm for $>1000$ noise realisations. The observables are extracted through averaging over the ensemble ${ }^{15,50}$. The initial state for the evolution of Eq. (1) is a thermal state obtained integrating Eq. (1) with constant $\epsilon$ fixed to its large initial value, for an integration time $>1000 \tau_{0}$ to ensure thermalisation. The temperature quench of the thermal cloud is simulated through a ramp in the chemical potential $(-\epsilon)$, keeping the noise amplitude $T$ and the damping $\gamma$ constant. This approximation captures the essential universal aspects of the dynamics. The symmetry breaking underlying the transition is induced by the change in $\epsilon$ (see the discussion following Eq. 1). Changes in $T$ and $\gamma$ give only non-universal quantitative corrections ${ }^{7,13}$. A similar method was successfully used in Ref. ${ }^{15}$ in the framework of the stochastic (projected) GrossPitaevskii equation (SPGPE) to model evaporative cooling of a pancake shaped thermal cloud and study the statistics of trapped vortices and found good agreement between experimental and numerical results. The SGPE and the SPGPE have been used before to model experimental results with success ${ }^{15,35,36,47-49}$.

To make direct connection of our simulation with experiments, we note that the one dimensional approximation of a thin 3D torus with thickness $d(d \ll \xi)$ is obtained by integrating out the transverse ( $y$ and $z$ in our case) degrees of freedom. Such a confinement-induced quasi-one dimensional Bose system is equivalent to a purely one-dimensional system with a modified interaction strength $\tilde{g}$ given by the confinement frequencies. For isotropic harmonic confinement in the transverse direction with confinement frequency $\omega_{0}$ we have $\tilde{g}=2 \hbar^{2} a / m a_{0}^{2}$, where $m$ is the mass of the atom, $a$ is the scattering length, and $a_{0}=\sqrt{\hbar / m \omega_{0}}$. The three dimensional wave function $\phi(x, y, z)$ may be reconstructed from the one-dimensional wave function $\phi(x)$ by assuming Gaussian profile in the transverse directions: $\phi(x, y, z)=\phi(x) \phi_{0}(y$, $z)$, where $\phi_{0}(y, z)=\frac{1}{\sqrt{a_{0} \pi}} e^{\left(y^{2}+z^{2}\right) / 2 a_{0}^{2}}$. Such reconstructions are shown in Fig. 1 and in the Supplementary Movie. The Supplementary Movie shows the real-time dynamics of the condensation process illustrating the key features of the dynamics germane to verification of KZM and determination of critical exponents, namely, stabilization of $W$ at the wake of the condensation and the knee-like behavior of the density growth (also clearly shown in the corresponding Supplementary Fig.). The simulation is done by integrating dimensionful SGPE with parameter values:

$a_{0}=100 \times a_{B}\left(a_{B}=\right.$ Bohr radius $), m=$ mass of a Rb atom, $\omega_{0}=200 \times 2 \pi, \gamma=0.01$ and $T=10 \mathrm{nK}$. All these parameters are compatible with the experimental set up in ${ }^{28,29}$

1. Kibble, T. W. B .Topology of cosmic domains and strings. J. Phys. A 9, 1387 (1976).

2. Kibble, T. W. B. Some implications of a cosmological phase transition. Phys. Rep. 67, 183-199 (1980).

3. Zurek, W. H. Cosmological experiments in superfluid helium? Nature 317, 505508 (1985).

4. Zurek, W. H. Cosmic strings in laboratory superfluids. Acta Phys. Pol. B 24, 1301 (1993).

5. Zurek, W. H. Cosmological experiments in condensed matter systems. Phys. Rep. 276, 177 (1996).

6. Zurek, W. H. Causality in condensates: gray solitons as relics of BEC formation Phys. Rev. Lett. 102, 105702 (2009).

7. Damski, B. \& Zurek, W. H. Soliton creation during a Bose-Einstein condensation. Phys. Rev. Lett. 104, 160404 (2010).

8. Witkowska, E., Deuar, P., Gajda, M. \& Rzazewski, M. Solitons as the early stage of quasicondensate formation during evaporative cooling. Phys. Rev. Lett 106, 135301 (2011).

9. Damski, B. \& Zurek, W. H. How to fix a broken symmetry: quantum dynamics of symmetry restoration in a ferromagnetic Bose-Einstein condensate. New J. Phys. 10, 045023 (2008).

10. Damski, B. \& Zurek, W. H. Quantum phase transition in space in a ferromagnetic spin-1 Bose-Einstein condensate. New J. Phys. 11, 063014 (2009).

11. Dziarmaga, J. Dynamics of a quantum phase transition and relaxation to a steady state. Adv. Phys. 59, 1063-1189 (2010).

12. Laguna, P. \& Zurek, W. H. Density of kinks after a quench: when symmetry breaks, how big are the pieces? Phys. Rev. Lett. 78, 2519 (1997).

13. Laguna, P. \& Zurek, W. H. Critical dynamics of symmetry breaking: quenches, dissipation, and cosmology. Phys. Rev. D 58, 085021 (1998).

14. del Campo, A., De Chiara, G., Morigi, G., Plenio, M. B. \& Retzker, A. Structural defects in ion chains by quenching the external potential: the inhomogeneous Kibble-Zurek mechanism. Phys. Rev. Lett. 105, 075701 (2010).

15. Weiler, C. N., Neely, T. W., Scherer, D. R., Bradley, A. S., Davis, M. J. \& Anderson, P. B. Spontaneous vortices in the formation of Bose-Einstein condensates. Nature 455, 948-951 (2008).

16. Scherer, D. R., Weiler, C. N., Neely, T. W. \& Anderson, P. B. Vortex Formation by Merging of Multiple Trapped Bose-Einstein Condensates. Phys. Rev. Lett. 98, 110402 (2007).

17. Sadler, L. E., Higbie, J. M., Leslie, S. R., Vengalattore, M. \& Stamper-Kurn, D. M. Spontaneous symmetry breaking in a quenched ferromagnetic spinor BoseEinstein condensate. Nature 443, 312-315 (2006).

18. Maniv, A., Polturak, E. \& Koren, G. Observation of magnetic flux generated spontaneously during a rapid quench of superconducting films. Phys. Rev. Lett. 91, 197001 (2003). 
19. Monaco, R., Mygind, J., Aaroe, M., Rivers, R. J. \& Koshelets, V. P. Zurek-Kibble mechanism for the spontaneous vortex formation in $\mathrm{Nb}-\mathrm{Al} / \mathrm{Alox} / \mathrm{Nb}$ Josephson tunnel junctions: new theory and experiment. Phys. Rev. Lett. 96, 180604 (2006).

20. Bauerle, C., Bunkov, Bauerle, C., Bunkov, Yu, M., Fisher, S. N.. Godfrin, H. \& Pickett, G. R. Laboratory simulation of cosmic string formation in the early Universe using superfluid 3He. Nature 382, 332-334 (1996).

21. Ruutu, V. M. H. et al. Vortex formation in neutron-irradiated superfluid $3 \mathrm{He}$ as an analogue of cosmological defect formation. Nature 382, 334-336 (1996).

22. Golubchik, D., Polturak, E. \& Koren G. Evidence for long-range correlations within arrays of spontaneously created magnetic vortices in a $\mathrm{Nb}$ thin-film superconductor. Phys. Rev. Lett. 104, 247002 (2010).

23. Monaco, R., Mygind, J., Rivers, R. J. \& Koshelets, V. P. Spontaneous fluxoid formation in superconducting loops. Phys. Rev. B 80, 180501(R) (2009).

24. Monaco, R., Mygind, J. \& Rivers, R. J. Zurek-Kibble domain structures: the dynamics of spontaneous vortex formation in annular Josephson tunnel junctions. Phys. Rev. Lett. 89, 080603 (2002).

25. Monaco, R., Mygind, J. \& Rivers, R. J. Spontaneous fluxon formation in annular Josephson tunnel junctions. Phys Rev. B 67, 104506 (2003).

26. Ueda, M., Kawaguchi, Y., Saito, Kanamoto, H. R. \& Nakajima, T. Symmetry breaking in Bose-Einstein condensates. AIP Conf. Proc. 869, 165 (2006).

27. Ryu, C. et al. Observation of Persistent Flow of a Bose-Einstein Condensate in a Toroidal Trap. Phys. Rev. Lett. 99, 260401 (2007).

28. Henderson, K., Ryu, C., MacCormick, C. \& Boshier, M. G. Experimental demonstration of painting arbitrary and dynamic potentials for Bose-Einstein condensates. New J Phys. 11, 043030 (2009).

29. Research Highlight of 28, Quantum physics: atomic painting. Nature 459, 142 (2009).

30. Gupta, S., Murch, K. W., Moore, K. L., Purdy, T. P. \& Stamper-Kurn, D. M. BoseEinstein condensation in a circular waveguide. Phys. Rev. Lett. 95, 143201 (2005).

31. Görlitz, A. et al. Realization of Bose-Einstein Condensates in lower dimensions. Phys. Rev. Lett. 87, 130402 (2001).

32. Köhl, M., Davis, M. J., Gardiner, C. W., Hänsch, T. W. \& Esslinger, T. Growth of Bose-Einstein condensates from thermal vapor. Phys. Rev. Lett. 88, 080402 (2002).

33. Ramanathan, A. et al. Superflow in a Toroidal Bose-Einstein Condensate: An Atom Circuit with a Tunable Weak Link. Phys. Rev. Lett. 106, 130401 (2011).

34. Sherlock, B. E., Gildemeister, M., Owen, E., Nugent, E. \& Foot, C. J. Time-averaged adiabatic ring potential for ultracold atoms arXiv:1102.2895v3 (2011).

35. Blakie, P. B., Bradley, A. S., Davis, M. J., Ballagh, R. J. \& Gardiner, C. W. Dynamics and statistical mechanics of ultra-cold Bose gases using c-field techniques. Adv. Phys. 57, 363-455 (2008).

36. Cockburn, S. P. \& Proukakis, N. P. The stochastic Gross-Pitaevskii equation and some applications. Laser Phys. 19, 558-570 (2009).

37. Stoof, H. T. C. \& Bijlsma, M. J. Dynamics of Fluctuating Bose-Einstein Condensates. J. Low Temp. Phys. 124, 431-442 (2001).

38. Saito, H., Kawaguchi, Y. \& Ueda, M. Kibble-Zurek mechanism in a quenched ferromagnetic Bose-Einstein condensate. Phys. Rev. A 76, 043613 (2007).

39. Dziarmaga, J., Meisner, J. \& Zurek, W. H. Winding up of the wave-function phase by an insulator-to-superfluid transition in a ring of coupled Bose-Einstein condensates. Phys. Rev. Lett. 101, 115701 (2008).
40. Reppy, J. D. \& Depatie, D. Persistent Currents in Superfluid Helium. Phys. Rev. Lett. 12187 (1964).

41. Antunes, N. D., Bettencourt, L. M. A. \& Zurek, W. H. Vortex string formation in a $3 \mathrm{D} \mathrm{U}(1)$ temperature quench. Phys. Rev. Lett. 82, 2824 (1999).

42. Damski, B. \& Zurek, W. H. Dynamics of a Quantum Phase Transition in a Ferromagnetic Bose-Einstein Condensate. Phys. Rev. Lett. 99, 130402 (2007).

43. Lythe, G. Stochastic PDEs: Domain Formation in Dynamic Transitions. Anales de Física 4, 55-63 (1998).

44. Donner, T. et al. Critical Behavior of a Trapped Interacting Bose Gas. Science $\mathbf{3 1 5}$, 1556-1558 (2007).

45. Kibble, T. W. B. \& Volovik, G. E. On phase ordering behind the propagating front of a second-order transition. JETP Lett. 65, 102-107 (1997).

46. Dziarmaga, J., Laguna, P. \& Zurek, W. H. Symmetry breaking with a slant: Topological defects after an inhomogeneous quench. Phys. Rev. Lett. 82, 4749 (1999).

47. Bradley, A. S., Gardiner, C. W. \& Davis, M. J. Bose-Einstein condensation from a rotating thermal cloud: Vortex nucleation and lattice formation. Phys. Rev. A 77, 033616 (2008).

48. Gardiner, C. W., Anglin, J. R. \& Fudge, T. I. A. The stochastic Gross-Pitaevskii equation. J. Phys. B 35, 1555 (2002).

49. Cockburn, S., Gallucci, D. \& Proukakis N. Quantitative study of quasi-onedimensional Bose gas experiments via the stochastic Gross-Pitaevskii equation. Phys. Rev. A 84, 023613 (2011).

50. Davis, M. J. \& Gardiner, C. Growth of a Bose-Einstein condensate: a detailed comparison of theory and experiment. J. Phys. B 35, 733 (2002).

\section{Acknowledgements}

We acknowledge support of U.S. Department of Energy through the LANL/LDRD Program. J.S. acknowledges support of Australian Research Council through the ARC Centre of Excellence for Quantum-Atom Optics. We thank B. Damski and M.J. Davis for useful discussions.

\section{Author contribution}

All authors contributed to the theoretical analysis, interpretation of the numerical data and the preparation of the manuscript. AD and JS carried out the numerical simulations. WHZ proposed and directed the project.

\section{Additional information}

Supplementary information accompanies this paper at http://www.nature.com/ scientificreports

Competing financial interests: The authors declare no competing financial interests. License: This work is licensed under a Creative Commons Attribution-NonCommercial-NoDerivative Works 3.0 Unported License. To view a copy of this license, visit http://creativecommons.org/licenses/by-nc-nd/3.0/

How to cite this article: Das, A., Sabbatini, J. \& Zurek, W.H. Winding up superfluid in torus via Bose Einstein condensation. Sci. Rep. 2, 352; DOI:10.1038/srep00352 (2012). 